\title{
CONVERGENCE TO STOCHASTIC INTEGRALS FOR DEPENDENT HETEROGENEOUS PROCESSES
}

\author{
BRUCE E. HANSEN \\ University of Rochester
}

This paper provides conditions to establish the weak convergence of stochastic integrals. The theorems are proved under the assumption that the innovations are strong mixing with uniformly bounded $2+$ moments. Several applications of the results are given, relevant for the theories of estimation with $I(1)$ processes, $I(2)$ processes, processes with nonstationary variances, nearintegrated processes, and continuous time approximations.

\section{INTRODUCTION}

A considerable research program has developed in econometrics concerning an asymptotic distribution theory for integrated and near-integrated processes. Frequently, the theory involves sequences of cadlag ${ }^{1}$ processes $\left\{U_{n}(s), V_{n}(s)\right\}$ which converge weakly in the Skorohod topology to $\{U(s), V(s)\}$. It is often desirable to obtain the limiting distribution of the integral process $\int_{0}^{s} U_{n} d V_{n}$. In certain cases, $\int_{0}^{s} U_{n} d V_{n} \Rightarrow \int_{0}^{s} U^{-} d V,{ }^{2}$ but it is known that this result is typically violated when $V_{n}$ is not a martingale. See, for example, Phillips [16].

There is a growing literature studying the asymptotic distribution of stochastic integrals. Chan and Wei [4, Lemma 2.4] derive the distribution of $\int_{0}^{1} U_{n} d V_{n}$ when $U_{n}$ and $V_{n}$ are martingale arrays with uniformly bounded conditional variances (which excludes many conditionally heteroskedastic processes, such as ARCH). Phillips [19] extends the results of [4] to allow the innovations to be general linear processes with independent identically distributed innovations. Strasser [22, Theorem 1.7] derives a functional limit theorem for $\int_{0}^{s} U_{n} d V_{n}$, for the case that $V_{n}$ is a martingale array and $U_{n}$ a Lipschitz function of a martingale array. In another paper, Phillips [18] attempts an alternative proof for mixing processes, but makes an error. ${ }^{3}$ Jeganathan [9, Proposition 6] provides a proof for the case that $V_{n}$ is a martingale array and $(U, V)$ are continuous processes. The most general results

This paper has grown out of work initiated in my Ph.D. thesis. I would like to thank Peter Phillips and Don Andrews for helpful advice, encouragement, and comments, and three referees for a careful reading of earlier drafts, which eliminated several important errors, led me to new references, and generally improved the quality of the paper. This research was partially supported by the NSF. 
yet published for martingale arrays are contained in Kurtz and Protter [10]. These authors consider a variety of limit theorems when $V_{n}$ is a semimartingale.

This paper provides an asymptotic theory for stochastic integrals which arise in econometric applications. The assumptions allow for weakly dependent heterogeneous data. Section 2 considers stochastic integrals with respect to a martingale process. Section 3 develops a martingale difference approximation for strong mixing sequences. Section 4 demonstrates the usefulness of the results for several examples of major interest. In all cases, the differences of $V_{n}$ are assumed to be strong mixing. The first example assumes that the differences of $U_{n}$ are strong mixing, which has applications in the theory of multivariate unit roots [4] and cointegration among $I(1)$ variables $[8,12]$. The second example assumes that $U_{n}$ is the product of processes whose differences are strong mixing, which has applications in the theory of heteroskedastic cointegration [6] and nonstationary variances [7]. The third example assumes that the second differences of $U_{n}$ are strong mixing, which has applications in the theory of cointegration among $I(2)$ variables [13]. The final example assumes that $U_{n}$ is a vector process with a root local to unity, which has applications in the theory of near-integration $[3,17,20]$ and continuous time approximations $[14,15]$. The appendix contains the proofs.

A word on notation. The symbol " $\equiv$ " denotes equality in distribution, $\|B\|_{r}=\left(\sum_{i j} E\left|B_{i j}\right|^{r}\right)^{1 / r}$ denotes the $L^{r}$-norm, "BM $(\Omega)$ " denotes vector Brownian motion with covariance matrix $\Omega, M^{k m}$ denotes the real-valued, $k \times m$ matrices, $D$ denotes the space of cadlag functions, and " $\Rightarrow$ " denotes weak convergence with respect to the Skorohod metric (as defined in [2]).

\section{MARTINGALE DIFFERENCES}

Consider random arrays $\left\{U_{n t}, Y_{n t}: 1 \leq t \leq n ; n \geq 1\right\}$ where $U_{n t}$ is a $k \times m$ matrix and $Y_{n t}$ is an $m \times 1$ vector. We can transform these arrays into random elements on $[0,1]$ by defining

$U_{n}(s)=U_{n[n s]}, \quad Y_{n}(s)=Y_{n[n s]}, \quad 0 \leq s \leq 1$.

Also, define the differences $\epsilon_{n t}=Y_{n t}-Y_{n, t-1}$. We can then define the stochastic integral

$\int_{0}^{s} U_{n}(r) d Y_{n}(r)=\int_{0}^{s} U_{n} d Y_{n}=\sum_{i=1}^{[n s]} U_{n i} \epsilon_{n i+1}$.

To evaluate the limit distribution of $\int_{0}^{s} U_{n} d Y_{n}$, we start by assuming that $Y_{n t}$ is a martingale. This allows us to apply a result from Kurtz and Protter [10] to obtain the following theorem. 
THEOREM 2.1. For each $n$, let $\left\{U_{n t}, Y_{n t}\right\}$ be an $\left\{\mathcal{F}_{n t}\right\}$-adapted process with sample paths in $\mathscr{D}_{M^{k m} \times \mathbb{R}^{m}}[0,1]$, and let $Y_{n t}$ be an $\left\{\mathcal{F}_{n t}\right\}$-martingale. If $\left(U_{n}, Y_{n}\right) \Rightarrow(U, Y)$ in $D_{M^{k m} \times \mathbb{R}^{m}}[0,1]$, and $\sup _{n} \sum_{i=1}^{n} E\left(\epsilon_{n i}^{2}\right)<\infty$, then $Y$ is a martingale with respect to a filtration to which $U$ and $Y$ are adapted, and

$$
\left(U_{n}, Y_{n}, \int U_{n} d Y_{n}\right) \Rightarrow\left(U, Y, \int U^{-} d Y\right) \text { in } D_{M^{k m} \times \mathbb{R}^{m} \times \mathbb{R}^{k}}[0,1] .
$$

Theorem 2.1 is a special case of one of the several theorems provided in Kurtz and Protter [10]. Their results encompass a broad range of processes, including semimartingales with weaker moment conditions. For the applications considered in this paper, however, the level of generality provided in Theorem 2.1 is sufficient.

\section{MIXING SEQUENCES}

Theorem 2.1 gives general conditions for the convergence of stochastic integrals when the process $\epsilon_{n i}$ is a square integrable martingale difference. This is not sufficiently general for many applications in econometrics. Phillips [19] used a martingale difference approximation taken from Hall and Heyde [5] to derive analogous results for strictly stationary linear processes. We extend this analysis to cover strong mixing ( $\alpha$-mixing) sequences.

To facilitate the analysis, we assume that the array $\left\{V_{n t}\right\}$ is a normalized stochastic partial sum process:

$V_{n t}=\frac{1}{\sqrt{n}} V_{t}, \quad V_{t}=\sum_{i=1}^{t} v_{i}$.

The stochastic integral of interest is

$$
\int_{0}^{s} U_{n} d V_{n}=\frac{1}{\sqrt{n}} \sum_{i=1}^{[n s]} U_{n i} v_{i+1}
$$

We assume that the sequence $\left\{v_{t}\right\}$ satisfies the following assumption.

Assumption 1. For some $p>\beta>2,\left\{v_{i}\right\}$ is a zero mean, strong mixing sequence with mixing coefficients $\alpha_{m}$ of size $-p \beta /(p-\beta)$ and $\sup _{i \geq 1}\left\|v_{i}\right\|_{p}=$ $C<\infty$. In addition, $(1 / n) E\left(V_{n} V_{n}^{\prime}\right) \rightarrow \Omega<\infty$ as $n \rightarrow \infty$.

Set $\mathcal{F}_{t}=\sigma\left(U_{n i}, v_{i}: i \leq t, n \geq 1\right\}$ to be the smallest sigma-field containing the past history of $\left\{U_{n t}, v_{t}\right\rangle$ for all $n$, and denote $E\left(X \mid \mathcal{F}_{i}\right)$ by $E_{i} X$. Define $\epsilon_{i}=\sum_{k=0}^{\infty}\left(E_{i} v_{i+k}-E_{i-1} v_{i+k}\right), \quad z_{i}=\sum_{k=1}^{\infty} E_{i} v_{i+k}$. 
It is not hard to verify that

$v_{i}=\epsilon_{i}+z_{i-1}-z_{i}, \quad E_{i-1} \epsilon_{i}=0$.

This representation is useful because of the equality

$\int_{0}^{s} U_{n} d V_{n}=\int_{0}^{s} U_{n} d Y_{n}+\Lambda_{n}^{*}(s)$

where $Y_{n}(s)=Y_{n[n s]}, Y_{n t}=Y_{t} / \sqrt{n}, Y_{t}=\sum_{1}^{t} \epsilon_{i}$, and

$\Lambda_{n t}^{*}=\frac{1}{\sqrt{n}} \sum_{i=1}^{t}\left(U_{n i}-U_{n i-1}\right) z_{i}^{\prime}-\frac{1}{\sqrt{n}} U_{n t} z_{t+1}^{\prime}$.

Since $\left\{\epsilon_{i}, \mathcal{F}_{i}\right\}$ is a martingale difference sequence, $\int_{0}^{s} U_{n} d Y_{n}$ can be analyzed via Theorem 2.1. This is stated in the following theorem.

THEOREM 3.1. If Assumption 1 holds and $\left(U_{n}, V_{n}\right) \Rightarrow(U, V)$ in $D_{M^{k m} \times \mathbb{R}^{m}}[0,1]$ then

$\int_{0}^{s} U_{n} d Y_{n} \Rightarrow \int_{0}^{s} U^{-} d V$

with $V(s)=\operatorname{BM}(\Omega)$, and $\Omega$ being defined in Assumption 1 .

Theorem 3.1 gives a convergence result for the martingale approximation $\int_{0}^{s} U_{n} d Y_{n}$. To achieve a convergence result for the process $\int_{0}^{s} U_{n} d V_{n}$ itself, it is necessary to examine the process $\Lambda_{n t}^{*}$. We have not been able to establish a general result. Instead, in the next section we illustrate the results for several common applications. We will find the following two results useful in the sequel. Theorem 3.2 gives a mixingale-type bound for the sequence $\left\{v_{i} z_{i}^{\prime}-E v_{i} z_{i}^{\prime}\right\}$. Theorem 3.3 shows that $L^{1}$-mixingales are asymptotically uncorrelated with arrays which have continuous asymptotic sample paths.

THEOREM 3.2. If Assumption 1 holds, then

$\left\|E_{i-m}\left(v_{i} z_{i}^{\prime}-E v_{i} z_{i}^{\prime}\right)\right\|_{\beta / 2} \leq C^{2} \psi_{m}$

where $\psi_{m}=12 m \alpha_{m}^{2(1 / \beta-1 / p)}+14 \sum_{k=m}^{\infty} \alpha_{k}^{2(1 / \beta-1 / p)}$, and $\Psi=\sum_{m=1}^{\infty} \psi_{m}<\infty$.

THEOREM 3.3. Suppose $U_{n} \Rightarrow U$ in $D_{M^{k m}}[0,1]$ and $U(\cdot)$ is almost surely continuous. For a random sequence $\left\{e_{i}\right\}$ and a sequence of nondecreasing sigma fields $\left\{\mathcal{F}_{i}^{e}\right\}$ to which $\left\{e_{i}\right\}$ is adapted, assume that $\sup _{i} E\left|E\left(e_{i} \mid \mathcal{F}_{i-m}^{e}\right)\right| \rightarrow$ 0 as $m \rightarrow \infty$. Then $\sup _{0 \leq s \leq 1}\left|\frac{1}{n} \sum_{i=1}^{[n s]} U_{n i} e_{i}\right| \vec{p} 0$. 
Theorem 3.3 has the following interesting corollary.

COROLLARY. For a random sequence $\left\{e_{i}\right\}$ and a sequence of nondecreasing sigma fields $\left\{\mathcal{F}_{i}^{e}\right\}$ to which $\left\{e_{i}\right\}$ is adapted, assume that $\sup _{i} E\left|E\left(e_{i} \mid \mathcal{F}_{i-m}^{e}\right)\right| \rightarrow 0$ as $m \rightarrow \infty$. Then $\max _{t \leq n}\left|\frac{1}{n} \sum_{i=1}^{t} e_{i}\right| \vec{p} 0$.

\section{APPLICATIONS}

In the following applications, $V_{n}$ is defined as in (1).

\section{1. /(1) Processes}

For our first application, we set $U_{n t}=V_{n t}$. Thus,

$\int_{0}^{s} U_{n} d V_{n}^{\prime}=\int_{0}^{s} V_{n} d V_{n}^{\prime}=\frac{1}{n} \sum_{i=1}^{[n s]} V_{i} v_{i+1}^{\prime}$.

Convergence of matrices of this form is an important component of regression theory under cointegration (see [12] for an application involving $\int_{0}^{1} V_{n} d V_{n}$, or [8] for an application using the stochastic integral process $\left.\int_{0}^{s} V_{n} d V_{n}^{\prime}\right)$. The only existing valid result which allows for serial correlation is [19] which uses general linear processes with square integrable i.i.d. innovations. This is quite restrictive, excluding, for example, conditional heteroskedasticity. The following theorem allows for strong mixing processes, and provides the first proof of weak convergence of the stochastic integral process for serially correlated arrays. Define

$\Lambda=\lim _{n \rightarrow \infty} \frac{1}{n} \sum_{i=1}^{n} \sum_{j=i+1}^{\infty} E\left(v_{i} v_{j}^{\prime}\right)$.

THEOREM 4.1. If Assumption 1 holds, then

$\int_{0}^{s} V_{n} d V_{n}^{\prime} \Rightarrow \int_{0}^{s} B d B^{\prime}+s \Lambda, \quad$ as $n \rightarrow \infty$,

where $B \equiv B M(\Omega)$.

\subsection{Products of /(1) Processes}

For our second application we set $U_{n t}=V_{n t} \otimes V_{n t}=(1 / n) V_{t} \otimes V_{t}$. Thus, $\int_{0}^{s} U_{n} d V_{n}^{\prime}=\int_{0}^{s}\left(V_{n} \otimes V_{n}\right) d V_{n}^{\prime}=\frac{1}{n^{3 / 2}} \sum_{i=1}^{[n s]}\left(V_{t} \otimes V_{t}\right) v_{t+1}^{\prime}$. 
Stochastic integrals of this form have arisen in models with nonstationary variances $[6,7]$.

THEOREM 4.2. If Assumption 1 holds with $\beta=3$, then

$$
\int_{0}^{s}\left(V_{n} \otimes V_{n}\right) d V_{n}^{\prime} \Rightarrow \int_{0}^{s}(B \otimes B) d B^{\prime}+\Lambda \otimes \int_{0}^{s} B+\int_{0}^{s} B \otimes \Lambda .
$$

\section{3. /(2) Processes}

The third application sets $U_{n t}=(1 / n) \sum_{i=1}^{t} V_{n i}=n^{-3 / 2} \bar{V}_{t}$, where $\bar{V}_{t}=$ $\sum_{i=1}^{t} V_{t}$. Thus,

$\int_{0}^{s} U_{n} d V_{n}^{\prime}=n^{-2} \sum_{i=1}^{[n s]} \bar{V}_{i} v_{i+1}^{\prime}$.

$\bar{V}_{i}$ is known as an $I(2)$ process. Stochastic integrals of this form are studied, for example, in [13].

THEOREM 4.3. If Assumption 1 holds, then

$\int_{0}^{s} U_{n} d V_{n}^{\prime} \Rightarrow \int_{0}^{s} \bar{B} d B^{\prime}, \quad$ as $n \rightarrow \infty$,

where $\bar{B}(r)=\int_{0}^{r} B$.

\subsection{Near-Integrated Processes}

The final application is to near-integrated arrays, which have been studied by [3], [17], and [20], and relate to continuous time approximations [14,15]. Define for some matrix $G$ the near-integrated array

$X_{n i}=\exp (G / n) X_{n i-1}+v_{i}$

and the limit diffusion

$U_{G}(r)=\int_{0}^{r} \exp \{(r-\lambda) G\} d B(\lambda)$.

Set $U_{n i}=(1 / \sqrt{n}) X_{n i}$. Here

$\int_{0}^{s} U_{n} d V_{n}^{\prime}=\frac{1}{n} \sum_{i=1}^{[n s]} X_{n i} v_{i}^{\prime}$.

THEOREM 4.4. If Assumption 1 holds, then

(a) $U_{n[n s]} \Rightarrow U_{G}(s)$

(b) $\int_{0}^{s} U_{n} d V_{n}^{\prime} \Rightarrow \int_{0}^{s} U_{G} d B^{\prime}+s \Lambda$. 


\section{NOTES}

1. A process is cadlag if it is right continuous with left limits.

2. $U^{-}(\cdot)$ is the left limit of $U(\cdot)$.

3. Equation (24) in the proof of Lemma 2.5 (b) in [18] is valid, but the subsequent critical step is invalid, for the processes $w_{t}$ and $L_{G}$ depend upon the matrix $G$.

\section{REFERENCES}

1. Andrews, D.W.K. Laws of large numbers for dependent non-identically distributed random variables. Econometric Theory 4 (1988): 458-467.

2. Billingsley, P. Convergence of Probability Measures. New York: Wiley, 1968.

3. Chan, N.H. \& C.Z. Wei. Asymptotic inference for nearly nonstationary AR(1) processes. Annals of Statistics 15 (1987): 1050-1063.

4. Chan, N.H. \& C.Z. Wei. Limiting distributions of least-squares estimates of unstable autoregressive processes. Annals of Statistics 16 (1988): 367-401.

5. Hall, P. \& C.C. Heyde. Martingale Limit Theory and Its Application. New York: Academic Press, 1980.

6. Hansen, B.E. Heteroskedastic cointegration. Journal of Econometrics (1992), forthcoming.

7. Hansen, B.E. Regression theory when variances are nonstationary. Unpublished manuscript, University of Rochester, 1990.

8. Hansen, B.E. Tests for parameter instability in regressions with I(1) processes. Journal of Business and Economic Statistics 10 (1992): 321-335.

9. Jeganathan, P. On the asymptotic behavior of least-squares estimators in AR time series models with roots near the unit circle. Econometric Theory 7 (1991): 269-306.

10. Kurtz, T.G. \& P. Protter. Weak limit theorems for stochastic integrals and stochastic differential equations. The Annals of Probability 19 (1991): 1035-1070.

11. McLeish, D.L. A maximal inequality and dependent strong laws. The Annals of Probability 3 (1975): 829-839.

12. Park, J.Y. \& P.C.B. Phillips. Statistical inference in regressions with integrated processes: Part 1. Econometric Theory 4 (1988): 468-497.

13. Park, J.Y. \& P.C.B. Phillips. Statistical inference in regressions with integrated processes: Part 2. Econometric Theory 5 (1989): 95-131.

14. Perron, P. A continuous time approximation to the first-order autoregressive process: The case without an intercept. Econometrica 59 (1991): 211-236.

15. Perron, P. A continuous time approximation to the stationary first-order autoregressive model. Econometric Theory (1991): 236-252.

16. Phillips, P.C.B. Time series regression with a unit root. Econometrica 55 (1987): 277-301.

17. Phillips, P.C.B. Towards a unified asymptotic theory for autoregression. Biometrika 74 (1987): 535-547.

18. Phillips, P.C.B. Weak convergence to the matrix stochastic integral $\int_{0}^{1} B d B^{\prime}$. Journal of Multivariate Analysis 24 (1988): 252-264.

19. Phillips, P.C.B. Weak convergence of sample covariance matrices to stochastic integrals via martingale approximations. Econometric Theory 4 (1988): 528-533.

20. Phillips, P.C.B. Regression theory for near-integrated time series. Econometrica 56 (1988): 1021-1044.

21. Protter, P. Stochastic Integration and Differential Equations: A New Approach. New York: Springer-Verlag, 1990.

22. Strasser, H. Martingale difference arrays and stochastic integrals. Probability Theory and Related Fields 72 (1986): 83-98.

23. Wooldridge, J.M. \& H. White. Some invariance principles and central limit theorems for dependent heterogeneous processes. Econometric Theory 4 (1988): 210-230. 


\section{APPENDIX}

Proof of Theorem 2.1. We simply need to verify the conditions of Theorem 4.6 of [10]. Since $Y_{n}$ is a martingale, it is a semimartingale, and their condition (4.20) holds trivially with $\gamma_{n}^{T}(\eta) \equiv 0$. To show that their condition C2.2(i) holds, set $\delta=\infty$ and $\tau_{n}^{a}=2 a$. Thus, for all $a>0, P\left\{\tau_{n}^{a} \leq a\right\}=0 \leq 1 / a$. In the notation of Kurtz-Protter, $M_{n}^{\delta}=Y_{n}^{\delta}=Y_{n}, A_{n}^{\delta}=0$, and

$$
\begin{array}{rl}
\sup _{n} & E\left(\left[M_{n}^{\delta}\right]_{s \Lambda \tau_{n}^{a}}+T_{s \Lambda \tau_{n}^{a}}\left(A_{n}^{\delta}\right)\right) \\
= & \sup _{n} E\left[Y_{n}\right]_{s \Lambda 2 a}=\sup _{n} E \sum_{1}^{[n(s \Lambda 2 a)]} \epsilon_{n i}^{2} \leq \sup _{n} E \sum_{1}^{n} \epsilon_{n i}^{2}<\infty .
\end{array}
$$

In this expression, $\left[Y_{n}\right]$ denotes the quadratic variation process of $Y_{n}$, and $T(A)$ denotes the total variation of $A$.

Proof of Theorem 3.1. We first establish the moment condition for $\epsilon_{n i}$. By Minkowski's inequality and McLeish's strong mixing inequality [11]

$$
\begin{aligned}
\left\|z_{l}\right\|_{\beta} & =\left\|\sum_{k=1}^{\infty} E_{i-1} v_{i+k}\right\|_{\beta} \leq \sum_{k=1}^{\infty}\left\|E_{i-1} v_{i+k}\right\|_{\beta} \\
& \leq \sum_{k=1}^{\infty} 6 \alpha_{k}^{1 / \beta-1 / p}\left\|v_{i+k}\right\|_{p} \leq 6 C \sum_{k=1}^{\infty} \alpha_{k}^{1 / \beta-1 / p}<\infty,
\end{aligned}
$$

uniformly in $i$. Set $\epsilon_{n i}=\epsilon_{i} / \sqrt{n}$. It follows that

$$
\begin{aligned}
\sum_{i=1}^{n} E \epsilon_{n i}^{2} & \leq \sup _{i \leq n} E \epsilon_{i}^{2}=\left(\sup _{i \leq n}\left\|v_{i}-z_{i-1}+z_{i}\right\|_{2}\right)^{2} \\
& \leq\left(\sup _{i \leq n}\left\|v_{i}\right\|_{\beta}+2 \sup _{i \leq n}\left\|z_{i}\right\|_{\beta}\right)^{2}<\infty .
\end{aligned}
$$

Note that $V_{n}(s) \Rightarrow V(s)=\mathrm{BM}(\Omega)$ as shown by Wooldridge and White [23, Corollary 4.2]. It remains to show that $\left(U_{n}, Y_{n}\right) \Rightarrow(U, V)$. Since $\left(U_{n}, Y_{n}\right)=\left(U_{n}, V_{n}\right)+\left(0,\left(Y_{n}-V_{n}\right)\right)$,

the result follows from the continuous mapping theorem since $\left(U_{n}, V_{n}\right) \Rightarrow(U, V)$ and $\left(Y_{n}-V_{n}\right) \Rightarrow 0$. We now show the latter. For all $\eta>0$,

$$
\begin{aligned}
P\left\{\sup _{i \leq n}\left|z_{i}\right|\right. & >\sqrt{n} \eta\} \leq \sum_{i=1}^{n} P\left\{\left|z_{i}\right|>\sqrt{n} \eta\right\} \\
& \leq \sum_{i=1}^{n} \frac{1}{\eta^{\beta} n^{\beta / 2}} E\left|z_{i}\right|^{\beta} \leq\left(\eta^{-1} 6 C \sum_{1}^{\infty} \alpha_{k}^{1 / \beta-1 / p}\right)^{\beta} n^{1-\beta / 2} \rightarrow 0
\end{aligned}
$$

since $\beta>2$. Therefore

$$
\sup _{t \leq n}\left|Y_{n t}-V_{n t}\right| \leq 2 \frac{1}{\sqrt{n}} \sup _{t \leq n}\left|z_{t}\right| \rightarrow \vec{p}
$$


This establishes that $\left(Y_{n}-V_{n}\right) \Rightarrow 0$ in the uniform metric, which implies convergence in the Skorohod metric as well (since the zero function is continuous). The conditions of Theorem 2.1 are satisfied and we conclude that

$$
\int_{0}^{s} U_{n} d Y_{n}^{\prime} \Rightarrow \int_{0}^{s} U^{-} d V^{\prime}
$$

Proof of Theorem 3.2. By Minkowski's inequality

$$
\begin{aligned}
\left\|E_{i-m}\left[v_{i} z_{i}^{\prime}-E v_{i} z_{i}^{\prime}\right]\right\|_{\beta / 2} & =\left\|\sum_{k=1}^{\infty}\left(E_{i-m} v_{i} v_{i+k}^{\prime}-E v_{i} v_{i+k}^{\prime}\right)\right\|_{\beta / 2} \\
& \leq \sum_{k=1}^{m}\left\|E_{i-m} v_{i} v_{i+k}^{\prime}-E v_{i} v_{i+k}^{\prime}\right\|_{\beta / 2} \\
& +\sum_{k=m}^{\infty}\left(\left\|E_{i-m} v_{i} v_{i+k}^{\prime}\right\|_{p / 2}+E v_{i} v_{i+k}^{\prime} \|_{\beta / 2}\right) .
\end{aligned}
$$

By McLeish's strong mixing inequality [11], Minkowski's inequality, the Rao-Blackwell theorem, and Holder's inequality

$$
\begin{aligned}
\sum_{k=1}^{m} & \left\|E_{i-m} v_{i} v_{i+k}^{\prime}-E v_{i} v_{i+k}^{\prime}\right\|_{\beta / 2} \\
& \leq 6 m \alpha_{m}^{2 / \beta-2 / p}\left\|v_{i} v_{i+k}^{\prime}-E v_{i} v_{i+k}^{\prime}\right\|_{p / 2} \\
& \leq 6 m \alpha_{m}^{2 / \beta-2 / p}\left(\left\|v_{i} v_{i+k}^{\prime}\right\|_{p / 2}+\left\|E v_{i} v_{i+k}^{\prime}\right\|_{p / 2}\right) \\
& \leq 12 m \alpha_{m}^{2 / \beta-2 / p}\left\|v_{i}\right\|_{p}\left\|v_{i+k}\right\|_{p} \leq 12 C^{2} m \alpha_{m}^{2 / \beta-2 / p} .
\end{aligned}
$$

In addition,

$$
\begin{aligned}
\left\|E_{i-m} v_{i} v_{i+k}^{\prime}\right\|_{\beta / 2} & \leq\left\|v_{i} E_{i} v_{i+k}^{\prime}\right\|_{\beta / 2} \\
& \leq\left\|v_{i}\right\|_{p}\left\|E_{i} v_{i+k}\right\|_{\beta p /(2 p-\beta)} \leq 6 C^{2} \alpha_{k}^{2 / \beta-2 / p} .
\end{aligned}
$$

Finally, by an $\alpha$-mixing inequality [5, Corollary A.2],

$$
\left\|E v_{i} v_{i+k}^{\prime}\right\|_{\beta / 2} \leq 8 \alpha_{k}^{2 / \beta-2 / p}\left\|v_{i} v_{i+k}^{\prime}\right\|_{p / 2} \leq 8 C^{2} \alpha_{k}^{2 / \beta-2 / p} \text {. }
$$

(A.4), (A.5), (A.6), and (A.7) together establish the main result. Finally,

$$
\begin{aligned}
\sum_{m=1}^{\infty} \psi_{m} & =\sum_{m=1}^{\infty}\left(12 m \alpha_{m}^{2 / \beta-2 / p}+14 \sum_{k=m}^{\infty} \alpha_{k}^{2 / \beta-2 / p}\right) \\
& =26 \sum_{m=1}^{\infty} m \alpha_{m}^{2(1 / \beta-1 / p)}<\infty,
\end{aligned}
$$

since $\alpha_{m}^{1 / \beta-1 / p}$ is of size -1 by Assumption 1 .

Proof of Theorem 3.3. For some $\delta \in(0,1)$, set $N=[1 / \delta], t_{k}=[k n / N]+1$, and $t_{k}^{*}=t_{k+1}-1, N_{s}^{*}=[(N-1) s]$, and $t_{s k}^{*}=\min \left(t_{k}^{*},[n s]\right)$. Then 


$$
\begin{aligned}
\sup _{0 \leq s \leq 1}\left|\frac{1}{n} \sum_{i=1}^{[n s]} U_{n i} e_{i}\right| & =\sup _{0 \leq s \leq 1}\left|\frac{1}{n} \sum_{k=0}^{N_{s}^{*}} \sum_{t=t_{k}}^{t_{s k}^{*}} U_{n t} e_{t}\right| \\
& \leq \sup _{0 \leq s \leq 1}\left|\frac{1}{n} \sum_{k=0}^{N_{s}^{*}} U_{n t_{k}} \sum_{t=t_{k}}^{t_{s k}^{*}} e_{t}\right|+\sup _{0 \leq s \leq 1}\left|\frac{1}{n} \sum_{k=0}^{N_{s}^{*}} \sum_{t=t_{k}}^{t_{s k}^{*}}\left(U_{n t}-U_{t_{k}}\right) e_{t}\right| \\
& \leq \frac{1}{n} \sum_{k=0}^{N-1}\left|U_{n t_{k}}\right|\left|\sum_{t=t_{k}}^{t_{k}^{*}} e_{t}\right|+\frac{1}{n} \sum_{k=0}^{N-1} \sum_{t=t_{k}}^{t_{k}^{*}}\left|U_{n t}-U_{t_{k}}\right|\left|e_{t}\right| \\
& \leq \sup _{0 \leq s \leq 1}\left|U_{n}(s)\right| \frac{1}{n} \sum_{k=0}^{N-1}\left|\sum_{t=t_{k}}^{t_{k}^{*}} e_{t}\right| \\
& +\sup _{|r-s| \leq \delta}\left|U_{n}(r)-U_{n}(s)\right| \frac{1}{n} \sum_{i=1}^{n}\left|e_{i}\right| .
\end{aligned}
$$

Since $U_{n}$ converges in the Skorohod topology, $\sup _{s}\left|U_{n}(s)\right|=O_{p}(1)$, while $(1 / n) \times$ $\sum_{1}^{n}\left|e_{i}\right|=O_{p}(1)$ since $e_{i}$ is uniformly integrable. Furthermore,

$E \frac{1}{n} \sum_{k=0}^{N-1}\left|\sum_{t=t_{k}}^{t_{k}^{*}} e_{t}\right| \leq \frac{N}{n} \sup _{0 \leq k \leq N-1} E\left|\sum_{t=t_{k}}^{t_{k}^{*}} e_{t}\right| \leq \sup _{t \leq n} E\left|\frac{1}{\delta n} \sum_{i=t}^{t+\delta n} e_{i}\right| \rightarrow 0$

as $n \rightarrow \infty$, by Andrews [1, Theorem 1]. The proof is completed by noting that

$$
\begin{array}{cl}
\sup _{|r-s| \leq \delta}\left|U_{n}(r)-U_{n}(s)\right| \underset{d}{\vec{d}} \sup _{|r-s| \leq \delta}|U(r)-U(s)| & \text { as } n \rightarrow \infty, \\
\vec{p} 0 & \text { as } \delta \rightarrow 0,
\end{array}
$$

since $U(\cdot)$ is continuous.

Proof of Theorem 4.1. As discussed in the proof of Theorem 3.1,

$U_{n}(s)=V_{n}(s)=\frac{1}{\sqrt{n}} \sum_{1}^{[n s]} v_{i} \Rightarrow B(s) \equiv \operatorname{BM}(\Omega)$.

The martingale difference approximation (2) allows decomposition (3), which in this case is

$$
\int_{0}^{s} V_{n} d V_{n}^{\prime}=\int_{0}^{s} V_{n} d Y_{n}^{\prime}+\Lambda_{n[n]}^{*}
$$

with

$\Lambda_{n t}^{*}=\frac{1}{n} \sum_{i=1}^{t} v_{i} z_{i}^{\prime}-\frac{1}{n} V_{t} z_{t+1}^{\prime}$

By Theorem 3.1, $\int_{0}^{s} V_{n} d Y_{n}^{\prime} \Rightarrow \int_{0}^{s} B d B^{\prime}$. It remains to show that $\Lambda_{n[n s]}^{*} \Rightarrow s \Lambda$. Since the limit process is nonrandom, this is equivalent to uniform convergence in probability. It is therefore sufficient to consider the case where $\Lambda_{n t}^{*}$ is scalar.

First, observe that

$$
\sup _{t \leq n} \frac{1}{n}\left|V_{t} z_{t+1}\right| \leq \sup _{t \leq n}\left|\frac{1}{\sqrt{n}} V_{t}\right| \frac{1}{\sqrt{n}} \sup _{t \leq n}\left|z_{t}\right| \vec{p} 0
$$

since $\sup _{t \leq n}\left|V_{t}\right|=O_{p}(\sqrt{n})$ and $(1 / \sqrt{n}) \sup _{t \leq n}\left|z_{t}\right| \underset{p}{\overrightarrow{0}} 0$ as in (A3). 
Second, by Theorem 3.2, the sequence $\left\{v_{i} z_{i}^{\prime}-E v_{i} z_{i}^{\prime}\right\}$ is an $L_{\beta / 2}$-mixingale. Since $\beta>2$, the sequence is therefore a uniformly integrable $L_{1}$-mixingale. Applying the Corollary to Theorem 3.3, we find

$\sup _{t \leq n}\left|\frac{1}{n} \sum_{i=1}^{t}\left(v_{i} z_{i}-E v_{i} z_{i}\right)\right| \vec{p} 0$.

Finally, observe that

$E \frac{1}{n} \sum_{i=1}^{[n s]} v_{i} z_{i}^{\prime}=\frac{1}{n} \sum_{i=1}^{[n s]} E\left(v_{i} \sum_{k=1}^{\infty} E_{i} v_{i+k}^{\prime}\right)=\frac{1}{n} \sum_{i=1}^{[n s]} \sum_{k=1}^{\infty} E\left(v_{i} v_{i+k}^{\prime}\right) \underset{n \rightarrow \infty}{\rightarrow} s \Lambda$.

Proof of Theorem 4.2. By the continuous mapping theorem, $U_{n}=V_{n} \otimes V_{n} \Rightarrow$ $V \otimes V$. Employing the martingale difference approximation (2)-(3) and Theorem 3.1, we obtain

$\int_{0}^{s}\left(V_{n} \otimes V_{n}\right) d Y_{n}^{\prime} \Rightarrow \int_{0}^{s}(V \otimes V) d V^{\prime}$

To analyze the bias term $\Lambda_{n t}^{*}$, note that

$$
\begin{aligned}
\Lambda_{n t}^{*}= & \frac{1}{n^{3 / 2}} \sum_{i=1}^{t}\left(\left(V_{i} \otimes V_{i}\right)-\left(V_{i-1} \otimes V_{i-1}\right)\right) z_{i}^{\prime}+o_{p}(1) \\
= & \frac{1}{n^{3 / 2}} \sum_{i=1}^{t}\left(\left(V_{i-1} \otimes v_{i}\right)+\left(v_{i} \otimes V_{i-1}\right)+\left(v_{i} \otimes v_{i}\right)\right) z_{i}^{\prime}+o_{p}(1) \\
= & \frac{1}{n^{3 / 2}} \sum_{i=1}^{t} V_{i-1} \otimes\left(v_{i} z_{i}^{\prime}\right)+\frac{1}{n^{3 / 2}} \sum_{i=1}^{t}\left(v_{i} z_{i}^{\prime}\right) \otimes V_{i-1} \\
& +\frac{1}{n^{3 / 2}} \sum_{i=1}^{t}\left(v_{i} \otimes v_{i}\right) z_{i}^{\prime}+o_{p}(1) .
\end{aligned}
$$

First,

$E \sup _{t \leq n}\left|\frac{1}{n^{3 / 2}} \sum_{i=1}^{t}\left(v_{i} \otimes v_{i}\right) z_{i}^{\prime}\right| \leq \frac{1}{n^{3 / 2}} \sum_{i=1}^{n}\left\|v_{i}\right\|_{3}^{2}\left\|z_{i}\right\|_{3} \rightarrow 0$

as $n \rightarrow \infty$ by (A.1). Second, set $e_{i}=e_{i}=v_{i} z_{i}^{\prime}-\Lambda$, which by Theorem 3.2 satisfies the conditions of Theorem 3.3, yielding

$$
\sup _{0 \leq s \leq 1}\left|\frac{1}{n} \sum_{i=1}^{[n s]} V_{n i-1} \otimes e_{i}\right| \underset{p}{\rightarrow} 0
$$

and therefore

$$
\frac{1}{n^{3 / 2}} \sum_{i=1}^{[n s]} V_{i-1} \otimes\left(v_{i} z_{i}^{\prime}\right)=\frac{1}{n^{3 / 2}} \sum_{i=1}^{[n s]} V_{i-1} \otimes \Lambda+\frac{1}{n} \sum_{i=1}^{[n s]} V_{n i-1} \otimes e_{i} \Rightarrow \int_{0}^{s} B \otimes \Lambda
$$

by the continuous mapping theorem. Similarly,

$$
\frac{1}{n^{3 / 2}} \sum_{i=1}^{[n s]}\left(v_{i} z_{i}^{\prime}\right) \otimes V_{i-1} \Rightarrow \Lambda \otimes \int_{0}^{s} B
$$

(A.8)-(A.11) combine to yield the result. 
Proof of Theorem 4.3. Since $V_{n} \Rightarrow V$, it follows that $U_{n} \Rightarrow \bar{V}$ by the continuous mapping theorem. Therefore by Theorem 3.1,

$\int_{0}^{s} U_{n} d Y_{n}^{\prime} \Rightarrow \int_{0}^{s} \bar{V} d V^{\prime}$

Additionally, $\Lambda_{n[n s]}^{*} \Rightarrow 0$ since

$$
\begin{aligned}
\left\|\sup _{0 \leq s \leq 1} \Lambda_{n}^{*}(s)\right\|_{1} & =\left\|\sup _{0 \leq s \leq 1}\left|n^{-2} \sum_{i=1}^{[n s]} V_{i} z_{i+1}^{\prime}\right|\right\|_{1}+o_{p}(1) \\
& \leq n^{-2} \sum_{i=1}^{n}\left\|V_{i} z_{i+1}^{\prime}\right\|_{1}+o_{p}(1) \leq n^{-2} \sum_{i=1}^{n}\left\|V_{i}\right\|_{2}\left\|z_{i+1}^{\prime}\right\|_{2}+o_{p}(1) \\
& \rightarrow 0 \quad \text { as } n \rightarrow \infty .
\end{aligned}
$$

\section{Proof of Theorem 4.4}

(a) $U_{n[n s]}=\frac{1}{\sqrt{n}} \sum_{k=0}^{[n s]} \exp \left(-\left(\frac{[n s]-k}{n}\right) G\right) v_{k}^{\prime}+o_{p}(1)$

$$
=\exp \left(-\left(\frac{[n s]}{n}\right) G\right) \frac{1}{\sqrt{n}} \sum_{k=0}^{[n s]} \exp \left(\left(\frac{k}{n}\right) G\right)\left(\epsilon_{k}-z_{k-1}+z_{k}\right)+o_{p}(1) \text {. }
$$

Note that

$$
\begin{aligned}
& \exp \left(-\left(\frac{[n s]}{n}\right) G\right) \frac{1}{\sqrt{n}} \sum_{k=0}^{[n s]} \exp \left(\left(\frac{k}{n}\right) G\right) \epsilon_{k} \\
& \quad \Rightarrow \exp (-s G) \int_{0}^{s} \exp (\lambda G) d B(\lambda)^{\prime}=\int_{0}^{s} \exp (-(s-\lambda) G) d B(\lambda)^{\prime}=U_{G}(s)
\end{aligned}
$$

by Theorem 3.1. In additon, standard manipulations can show that

$$
\frac{1}{\sqrt{n}} \sum_{k=0}^{[n s]} \exp \left(\left(\frac{k}{n}\right) G\right)\left(z_{k}-z_{k-1}\right)^{\prime} \Rightarrow 0
$$

establishing the result.

(b) By Theorem 3.1, $\int_{0}^{s} U_{n} d Y_{n}^{\prime} \Rightarrow \int_{0}^{s} U_{G} d V$. In addition,

$$
\begin{aligned}
\Lambda_{n}^{*}(s) & =\frac{1}{n} \sum_{i=1}^{[n s]} \Delta X_{n i} z_{i}^{\prime}+o_{p}(1) \\
& =\frac{1}{n} \sum_{i=1}^{[n s]} v_{i} z_{i}^{\prime}+\left[\exp \left(-\frac{G}{n}\right)-I\right] \frac{1}{n} \sum_{i=1}^{[n s]} X_{n i-1} z_{i}^{\prime}+o_{p}(1) \Rightarrow s \Lambda
\end{aligned}
$$

as shown in the proof of Theorem 4.1. (Note that the $o_{p}(1)$ terms hold uniformly in $s \in[0,1]$.) 